\title{
A large active wave trapped in Jupiter's equator
}

\author{
J. Legarreta ${ }^{1,2}$, N. Barrado-Izagirre, ${ }^{2,3}$, E. García-Melendo ${ }^{2,4}$, A. Sánchez-Lavega ${ }^{2,3}$, \\ J. M. Gómez-Forrellad ${ }^{4}$, and the IOPW Team ${ }^{\star}$
}

\author{
${ }^{1}$ Departamento de Ingeniería de Sistemas y Automática, Universidad del País Vasco UPV/EHU, EUITI, \\ Paseo Rafael Moreno "Pitxitxi" 3, 48013 Bilbao, Spain \\ e-mail: jonjosu. legarreta@ehu.eus \\ 2 Unidad Asociada Grupo Ciencias Planetarias UPV/EHU-IAA(CSIC), 48013 Bilbao, Spain \\ 3 Departamento Física Aplicada I, Universidad del País Vasco UPV/EHU, ETS Ingeniería, Alameda Urquijo s/n, 48013 Bilbao, Spain \\ ${ }^{4}$ Fundació Observatori Esteve Duran, Montseny 46, 08553 Seva, Spain
}

Received 27 March 2015 / Accepted 9 December 2015

\begin{abstract}
Context. A peculiar atmospheric feature was observed in the equatorial zone (EZ) of Jupiter between September and December 2012 in ground-based and Hubble Space Telescope (HST) images. This feature consisted of two low albedo Y-shaped cloud structures ( $\mathrm{Y} 1$ and $\mathrm{Y} 2$ ) oriented along the equator and centred on it (latitude $0.5^{\circ}-1^{\circ} \mathrm{N}$ ).

Aims. We wanted to characterize these features, and also tried to find out their properties and understand their nature.

Methods. We tracked these features to obtain their velocity and analyse their cloud morphology and the interaction with their surroundings. We present numerical simulations of the phenomenon based on one- and two-layer shallow water models under a Gaussian pulse excitation.

Results. Each Y feature had a characteristic zonal length of $\sim 15^{\circ}(18000 \mathrm{~km})$ and a meridional width (distance between the northsouth extremes of the $\mathrm{Y})$ of $5^{\circ}(6000 \mathrm{~km})$, and moved eastward with a speed of around $20-40 \mathrm{~m} \mathrm{~s}^{-1}$ relative to Jupiter's mean flow. Their lifetime was 90 and 60 days for Y1 and Y2, respectively. In November, both Y1 and Y2 exhibited outbursts of rapidly evolving bright spots emerging from the Y vertex. The Y features were not visible at wavelengths of 255 or $890 \mathrm{~nm}$, which suggests that they were vertically shallow and placed in altitude between the upper equatorial hazes and the main cloud deck. Numerical simulations of the dynamics of the Jovian equatorial region generate Kelvin and Rossby waves, which are similar to those in the Matsuno-Gill model for Earth's equatorial dynamics, and reproduce the observed cloud morphology and the main properties the main properties of the $\mathrm{Y}$ features.
\end{abstract}

Key words. planets and satellites: atmospheres - planets and satellites: gaseous planets

\section{Introduction}

The equatorial region of Jupiter is dominated by an intense and broad eastward jet about $30^{\circ}$ wide in latitude (from $\sim 15^{\circ} \mathrm{N}$ to $15^{\circ} \mathrm{S}$ ) with two permanent velocity maximums at the north and south boundaries of the equatorial zone $\left(\mathrm{EZ}\right.$; at $\left.\pm 6^{\circ}\right)$ and a central minimum (Rogers 1995; Ingersoll et al. 2004; Limaye 1986; Simon 1999; García-Melendo \& Sánchez-Lavega 2001; Porco et al. 2003; Simon-Miller et al. 2007). The northern jet at $6^{\circ}-7^{\circ} \mathrm{N}$ is dominated by the presence of the North Equatorial Belt (NEB) plumes and bluish dark areas (seen as hot spots at 5 microns) with projections that have been interpreted as a Rossby wave (Allison 1990; Ortiz et al. 1998; Arregi et al. 2006). The velocity of this jet is difficult to separate from this wave motion and ranges from $\sim 100$ to $150 \mathrm{~m} \mathrm{~s}^{-1}$, depending on the plumes-wave activity (Asay-Davis et al. 2011; García-Melendo et al. 2011). In addition, the observed velocity ranges also seem to be related to the altitude of the cloud tracers and vertical wind shears ( $\mathrm{Li}$ et al. 2006; García-Melendo et al. 2011). The southern jet peaks at $6^{\circ} \mathrm{S}$, reaches velocities of around $140-150 \mathrm{~m} \mathrm{~s}^{-1}$ and its velocity seems to be less influenced by the Rossby wave activity, which is probably because of much greater

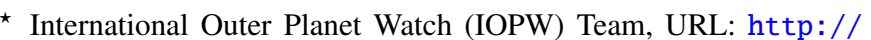
www. pvol.ehu.es/
}

wavenumbers and lower phase speeds than those in the north (García-Melendo et al. 2011; Simon-Miller et al. 2012). The central part of the equatorial jet has a minimum velocity of about 60 to $80 \mathrm{~m} \mathrm{~s}^{-1}$ at $0^{\circ}$ latitude and a parabolic shape between the north and south jets. Globally, Jupiter's equatorial jet can be seen as a symmetric double jet with maximum velocities of $\sim 150 \mathrm{~m} \mathrm{~s}^{-1}$ at the peaks and a central minimum with a velocity of $-70 \mathrm{~m} \mathrm{~s}^{-1}$ (westward) relative to the two jet peaks. This structure of the jet was proposed to be the result of a mixture of meridional motions related to Hadley cells and a Kelvin wave (Yamazaki et al. 2005).

The dark projections with base at $6^{\circ} \mathrm{N}$ extend towards the equator and form curved elongated features according to the meridional shear of the zonal wind. Occasionally they converge around latitude $0^{\circ}$ with similar but less contrasted thin projections from the south, forming the equatorial band, a slim dark belt in the middle of the EZ (Rogers 1995). High resolution images taken by spacecraft show a complex cloud morphology field and turbulent patterns in the middle of the EZ (García-Melendo et al. 2011). In addition, different types of short and large scale or planetary waves have been detected both in the thermal field and in the cloud field in the EZ. Gravity waves at cloud level travelling in the westward direction with wavelengths of about $300 \mathrm{~km}$ have been reported by different studies 
(Flasar \& Gierasch 1986; Reuter et al. 2007; Arregi et al. 2009). As stated above, large scale waves in the cloud field, pressumably of Rossby type, have been identified and studied by different teams (Allison 1990; Ortiz et al. 1998; Arregi et al. 2006; Li et al. 2006; Barrado-Izagirre et al. 2008; García-Melendo et al. 2011; Choi et al. 2013). Finally equatorial waves in the temperature field have been detected with wavenumbers in the range 1-11 (Deming et al. 1997). A summary of the properties of Jovian equatorial waves is listed in Table 8.4 in SánchezLavega et al. (2011).

In this paper, we report on the detection and study of a new kind of wave observed for a few months in 2012 on groundbased and HST images. The wave had a Y-like shape approximately symmetric with respect to the equator with its vertex aligned and centred on it. This kind of feature is different from the above described equatorial waves. Its aspect is reminiscent of the long-lived $\mathrm{Y}$ or $\mathrm{C}$ structure observed in Venus clouds (Schubert 1983) and recently identified as a wind distorted Kelvin wave by Peralta et al. (2015). We first describe the observations of the feature (Sect. 2). Section 3 is dedicated to the study of morphology, evolution and motions. In Sect. 4, we present measurements of the feature speed on the zonal wind. Finally, in Sect. 5, we present numerical simulations of the formation and structure of the Jovian equatorial waves based on a one- and two-layer shallow water model, which allows us to propose the Y wave to be a Rossby-Kelvin wave combination similar to that resulting in the Matsuno-Gill model for the Earth's atmophere (Matsuno 1966; Gill 1980).

\section{Observations}

This study is mainly based on the analysis of about 50 images submitted by several observers to the International Outer Planet Watch-Planetary Virtual Observatory (IOPW-PVOL) database (Hueso et al. 2010). The contributors and the telescopes employed are summarized in Table 1. The PVOL database stores more than 19000 images of Jupiter and Saturn submitted mainly by amateur astronomers from more than 30 countries $^{1}$. The quality and spatial resolution on these images depend on several parameters: planet size (date of the observations relative to Jupiter's opposition), atmospheric conditions (seeing), telescope diameter, camera used and processing software, planet altitude, and astronomer's location in latitude. Assuming $35 \mathrm{~cm}$ diameter as a representative size of telescope used, the Airy spot diameter at the yellow wavelength is 0.4 arcsec, which for a JupiterEarth distance $\left(\sim 6.26 \times 10^{8} \mathrm{~km}\right)$ gives a spatial resolution over Jupiter's disk of $\sim 1200 \mathrm{~km} / \mathrm{pixel}$; this is good enough to study Jovian atmospheric dynamics (Barrado-Izagirre et al. 2013).

Although with lower resolution when compared with visible images by the HST, the temporal coverage and sampling of the IOPW-PVOL images is much longer. The observations used in this work span four months (from September to December 2012) with at least daily image coverage. The two Y features studied in red giant branch (RGB) colour images were composed of individual red, green, and blue frames created using the lucky imaging technique (e.g. Law et al. 2006). In Fig. 1, we show the visual aspect of one of the features in three different days observed by different observers in the whole planet disc. The Y-like shape structure was more evident in October than in September or December. We selected the best images from that epoch to analyse morphology, evolution and motions of these features. In addition, we used sets of images obtained using the same lucky

\footnotetext{
http://www .pvol . ehu.es/pvol/
}

Table 1. IOPW/PVOL observations, observer, and instrumentation are described.

\begin{tabular}{|c|c|c|c|}
\hline Author & Location & $\begin{array}{c}\text { Telescope } \\
\text { aperture }(\mathrm{cm})\end{array}$ & Filter \\
\hline D. Parker & USA & 40.6 & RGB \\
\hline C. Pellier & France & 25 & RGB \\
\hline T. Barry & Australia & 40.6 & RGB \\
\hline T. Kumamori & Japan & 28 & LRGB \\
\hline T. Akutsu & Philippines & 35.5 & LRGB \\
\hline P. Edwards & UK & 28 & RGB \\
\hline J. Phillips & USA & 25.4 & RGB \\
\hline E. Morales & Puerto Rico & 30 & RGB \\
\hline J. Beltran & Spain & 45.7 & IR, RGB \\
\hline M. Mobberley & UK & 30 & RGB \\
\hline W. Jaeschke & USA & 35.6 & RGB \\
\hline S. Kidd & UK & 35.6 & RGB \\
\hline D. Tayler & UK & 35.6 & RGB \\
\hline C. Go & Philippines & 28 & RGB \\
\hline J. R. Sanchez & Spain & 19 & RGB \\
\hline C. Fatinnanzi & Italy & 36 & RGB,IR \\
\hline J.J. Poupeau & France & 35 & RGB, R \\
\hline D.P. Milika & Australia & 28 & RGB \\
\hline A. Lasala & Spain & 25.4 & LRGB,R \\
\hline I. Sharp & UK & 28 & RGB \\
\hline P. Casquinha & Portugal & 35.6 & RGB \\
\hline M. Delcroix & France & 25.4 & RGB, IR \\
\hline F. Willems & USA & 35.6 & RGB, IR \\
\hline M. Lecompte & UK & 35.5 & RGB, IR \\
\hline P. Maxson & USA & 25 & RGB \\
\hline D. Peach & UK & 35 & RGB \\
\hline G. Walker & USA & 25.4 & LRGB \\
\hline S. Walker & USA & 31.8 & RGB \\
\hline T. Wilson & USA & 25.4 & RGB \\
\hline
\end{tabular}

Notes. All of them are RGB composites (wavelength range from $\sim 400$ to $700 \mathrm{~nm}$ ) and the temporal coverage is of four months from 2012-0908 to $2012-12-13$.

imaging technique between September and December 2012 with a $28 \mathrm{~cm}$ telescope from the Aula EspaZio facility at the School of Engineers of the University of the Basque Country (UPV/EHU ${ }^{2}$; Mendikoa et al. 2012; Sánchez-Lavega et al. 2014; OrdóñezEtxeberria et al. 2014).

To complete the analysis of the phenomenon, we also used some images from the HST's public archive obtained with the Wide Field Planetary Camera 3 (WFC3). The spatial resolution of these images is a factor of 10 higher $\left(0.04^{\prime \prime} / \mathrm{pixel}\right)$ for the latitudes covered, which translates into about $150 \mathrm{~km} /$ pixel. The images we used were taken on September 20th at two wavelengths, in the red (Filter $F 763 M$, central wavelength $\sim 763 \mathrm{~nm}$ ) and in the ultraviolet (Filter $F 275 \mathrm{~W}$ at $275 \mathrm{~nm}$ ), and are listed in Table 2. These images were used to analyse the feature at high resolution and to measure the wind profile at two altitude levels: ammonia cloud (near infrared, $763 \mathrm{~nm}$ ) and the haze layer above it (UV, $275 \mathrm{~nm})$.

\section{Properties of the $\mathrm{Y}$ feature}

The EZ of Jupiter extends from latitudes $\sim 6^{\circ} \mathrm{S}$ to $6^{\circ} \mathrm{N}$ confined by the two symmetric equatorial eastward jets (García-Melendo et al. 2011). Because of the intense meridional shear of the zonal wind between these two jets, cloud features in the mid-EZ

\footnotetext{
2 http://www.ehu.eus/aula-espazio/imagenes_Jupiter. html
} 


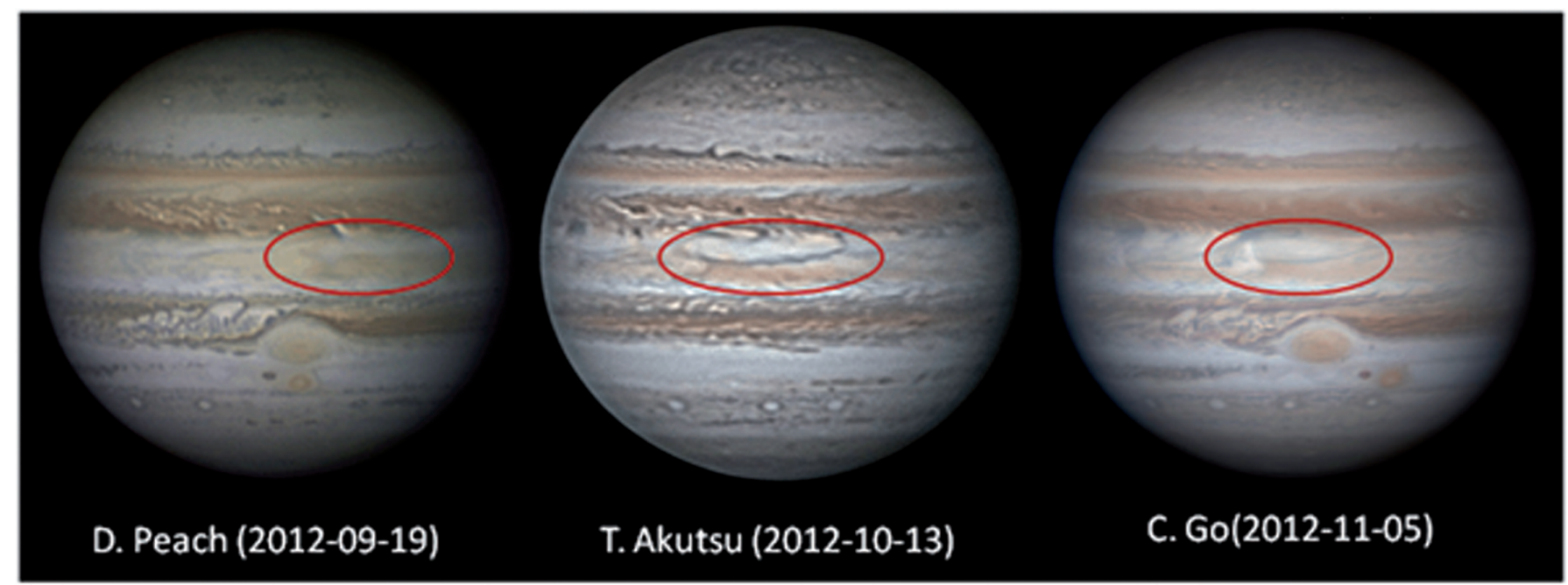

Fig. 1. Visual aspect of the first Y-like feature in three different days from IOPW-PVOL database. The orientation of all the figures in the paper is north up and east at right. Author and date are indicated.

Table 2. Log of the used HST archived images to retrieve Jupiter's zonal wind profile.

\begin{tabular}{cccc}
\hline \hline Date & Time & Image name & Filter \\
\hline Sept. 20th, 2012 & $01: 15: 19$ & IC3G02RYQ & $F 275 W$ \\
& $01: 19: 04$ & IC3G02RZO & $F 763 M$ \\
& $04: 26: 44$ & IC3G04SNQ & $F 275 W$ \\
& $04: 30: 29$ & IC3G04SOQ & $F 763 M$ \\
& $07: 38: 12$ & IC3G06TDQ & $F 275 W$ \\
& $07: 41: 57$ & IC3G06TEQ & $F 763 M$ \\
& $11: 04: 17$ & IC3G08TZQ & $F 275 W$ \\
& $11: 08: 02$ & IC3G08UOQ & $F 763 M$ \\
& $14: 23: 03$ & IC3G10UHQ & $F 275 W$ \\
& $14: 27: 21$ & IC3G10U1Q & $F 763 M$ \\
& $17: 34: 31$ & IC3G12V1Q & $F 275 W$ \\
& $17: 38: 16$ & IC3G12V2Q & $F 763 M$ \\
& $21: 04: 09$ & IC3G14VSQ & $F 275 W$ \\
& $21: 07: 54$ & IC3G14VTQ & $F 763 M$ \\
\hline
\end{tabular}

tend to be tilted from the north and south jet peaks towards the equator, where they join and form the Y-like shape features. Usually these patterns are unstable, and the $\mathrm{Y}$ - or C-arched shapes do not persist in time (Rogers 1995). In the last quarter of 2012, however, two Y-shaped structures formed and survived for months in the equator, but contrarily to what would be expected with the open side of the "Y" facing the minimum of the equatorial eastward jet (see bottom panel of Fig. 2). The first feature (Y1) formed in September at $1^{\circ} \mathrm{N}$ latitude and $300^{\circ}$ System III longitude. This feature moved eastward with a constant velocity until it vanished in December. Later in October, a second similar feature (Y2) appeared in the same latitude but at $100^{\circ}$ System III longitude.

We used LAIA software (Cano 1998) to analyse the morphology, motion, and evolution of the features. With LAIA we navigated the selected images providing a latitude and longitude value for each pixel of the image. We measured the features' location on images obtained along September-December 2012, typically with one image measure per day.

Figure 2 shows the morphology of Y1 in 19-20 September 2012 at different wavelengths. The Y-shaped structure appears as a low albedo feature centred in the EZ at visible wavelengths (RGB composites; IOPW) and at $763 \mathrm{~nm}$ red wavelengths in
HST and IOPW images. The feature does not stand out in the $890 \mathrm{~nm}$ methane absorption band (IOPW), where only a dark patch is observed. In the UV at $255 \mathrm{~nm}$ (HST) there is no sign of it at all. The methane and UV wavelengths are sensitive to the upper equatorial haze, and they show an inverse relative contrast at these two wavelengths. High features are bright in methane and dark in the UV. The fact that the Y is not bright (but dark) in methane and not visible in UV indicates that it is a relatively low altitude feature relative to the upper haze.

As has been mentioned, we tracked Y1 and Y2 features during more than three months (September to December 2012) retrieving their motions in latitude and longitude as shown in Fig. 3. Figure $3 \mathrm{a}$ shows that $\mathrm{Y} 1$ and $\mathrm{Y} 2$ followed a nearly perfect linear drift, which means a very stable and constant zonal velocity for both features: $99.4 \pm 0.1 \mathrm{~m} \mathrm{~s}^{-1}$ for Y1 and $101.4 \pm 0.6 \mathrm{~m} \mathrm{~s}^{-1}$ for $\mathrm{Y} 2$, both moving with a velocity above the average velocity measured for the area as we discuss later. Figure $3 b$ shows that $\mathrm{Y} 1$ and $\mathrm{Y} 2$ vertices remained at a fixed latitude, centred at $1^{\circ} \mathrm{N}$ and $0.5^{\circ} \mathrm{N}$, respectively.

In October and November we detected bright features (white spots) near the vertices of Y1 and Y2. As illustrated in Fig. 4, the first eruption (named St 1 ) appeared on October 31st on the west side of the Y1 vertex and since then there were many successive high albedo features at the Y1 and Y2 vertices. It is difficult to establish a lifetime for these bright phenomena, but it is of the order of two to four days (Fig. 5). Tracking the motion of these bright spots shows differences and coincidences with those of the $\mathrm{Y}$ features themselves. The first white spot had a velocity of $80 \mathrm{~m} \mathrm{~s}^{-1}$, moved slower than the Y1 feature, and migrated in latitude out of the equator (Fig. 5b). The other spots had the same velocity as the corresponding $\mathrm{Y}$ without any significant meridional drift; they moved all together suggesting that they were dynamically related features (Fig. 5).

We were able to measure the size of these spots (north-south and east-west limits) and its evolution (Fig. 6). For one of these spots (Stl) we detected that its area grew rapidly in a matter of four days (Fig. 6), suggesting that this could be due to horizontal expansion of the spot's cloud tops. Horizontal divergence of the velocity field is given by

$\operatorname{div}_{h} V=\nabla_{h} V=\frac{\partial u}{\partial x}+\frac{\partial v}{\partial y}=\frac{1}{A_{H}} \frac{\mathrm{d} A_{H}}{\mathrm{~d} t}$ 

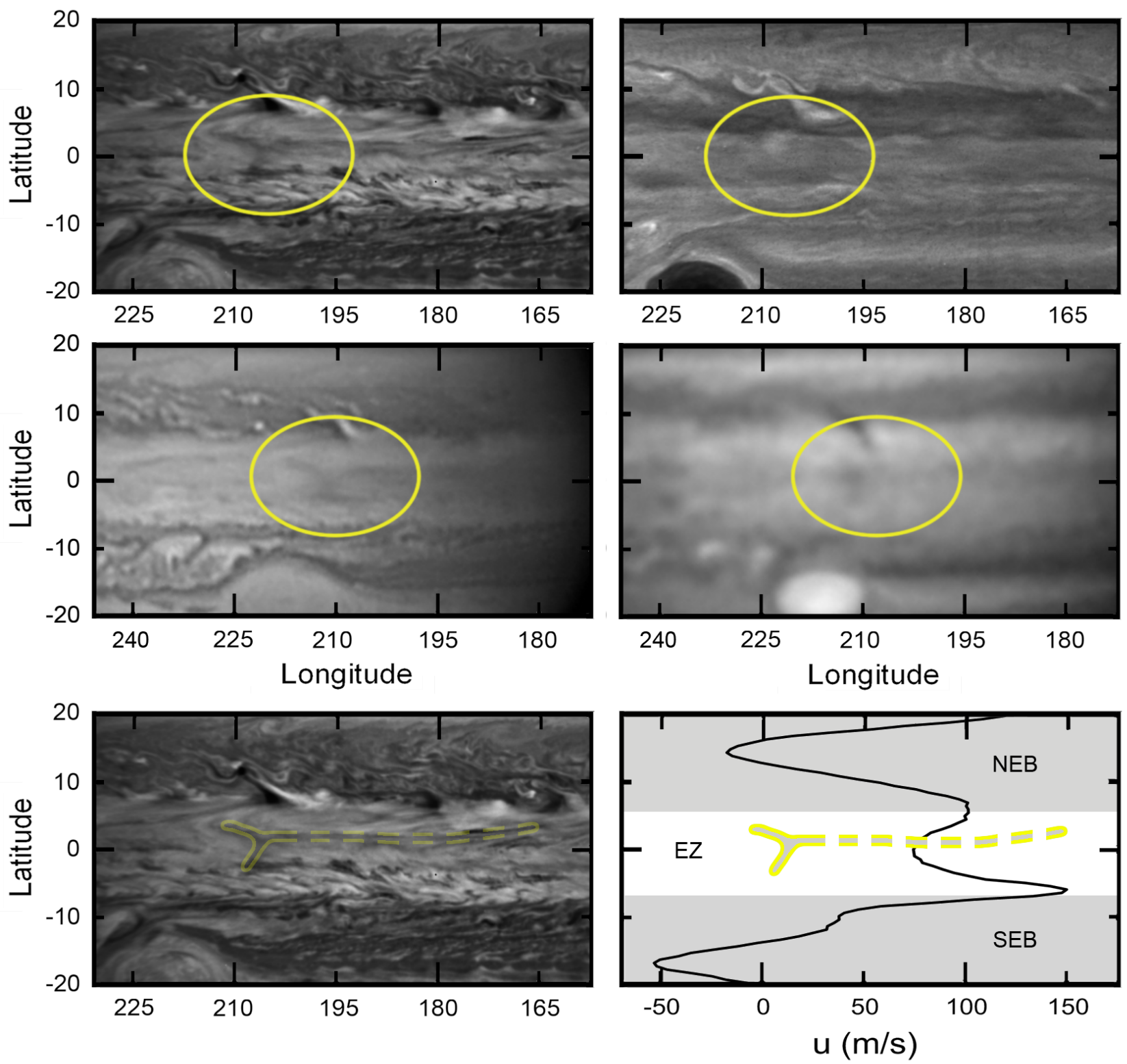

Fig. 2. Y-like feature's morphology. Top panels show two HST images (September 20th) taken the same day but in two distinct filters; the left image was taken with a NIR $(F 763 M)$ filter while the right image is observed by an UV $(F 275 W)$ filter. Middle panels show two images from the IOPW database by the same observer (D. Peach) taken on September 19th, left one in RGB and in methane absorption band the one on the right. In the bottom panel there is a cartoon representing where the Y-like feature is and where in the wind profile is it located. The Wind profile represented is the García-Melendo \& Sánchez-Lavega (2001) one (NEB: north equatorial Belt, EZ: equatorial zone and SEB: south equatorial belt).

where $A_{H}$ is the horizontal area of the eruption (Sánchez-Lavega et al. 2011). If this growing area is related to an updraft with vertical velocity $w$, mass conservation (continuity equation) implies that

$-\frac{\partial w}{\partial z}=\frac{\partial u}{\partial x}+\frac{\partial v}{\partial y}$

and assuming that the vertical updraft take place along a distance $h$, we obtain

$w \leqslant h \frac{\ln \left(A_{H 2} / A_{H 1}\right)}{\Delta t}$,

where $A_{H 1}$ and $A_{H 2}$ are the initial and final areas of the bright cloud measured in the interval $\Delta t$. From Fig. 6 , the area of the spot grew a factor 4 from 31 October to 3 November. The altitude $h$ is unknown but to first order, assuming altitude $h \sim$ scale height, $H=20 \mathrm{~km}$, we obtain a vertical velocity of $\sim 0.1 \mathrm{~m} \mathrm{~s}^{-1}$ (with an error of 25\%). This value is a factor 10 lower than those measured in rapidly expanding storms in Jupiter's major disturbances (Sánchez-Lavega \& Gómez 1996; Sánchez-Lavega et al. 2008) and lower than those predicted by moist convective models (Hueso \& Sánchez-Lavega 2001). It, therefore, seems that another mechanism, related to the Y-like feature and not so vigorous as convection, could produce the up-drafts.

\section{Speed of the $Y$ features relative to the zonal wind}

We have compared the motion of the $\mathrm{Y}$ features with that of the average zonal wind in the equatorial region. The meridional profile of the zonal wind was measured with two procedures. On the one hand, we used a two-dimensional automatic brightness correlation technique (Hueso et al. 2009) based on an 

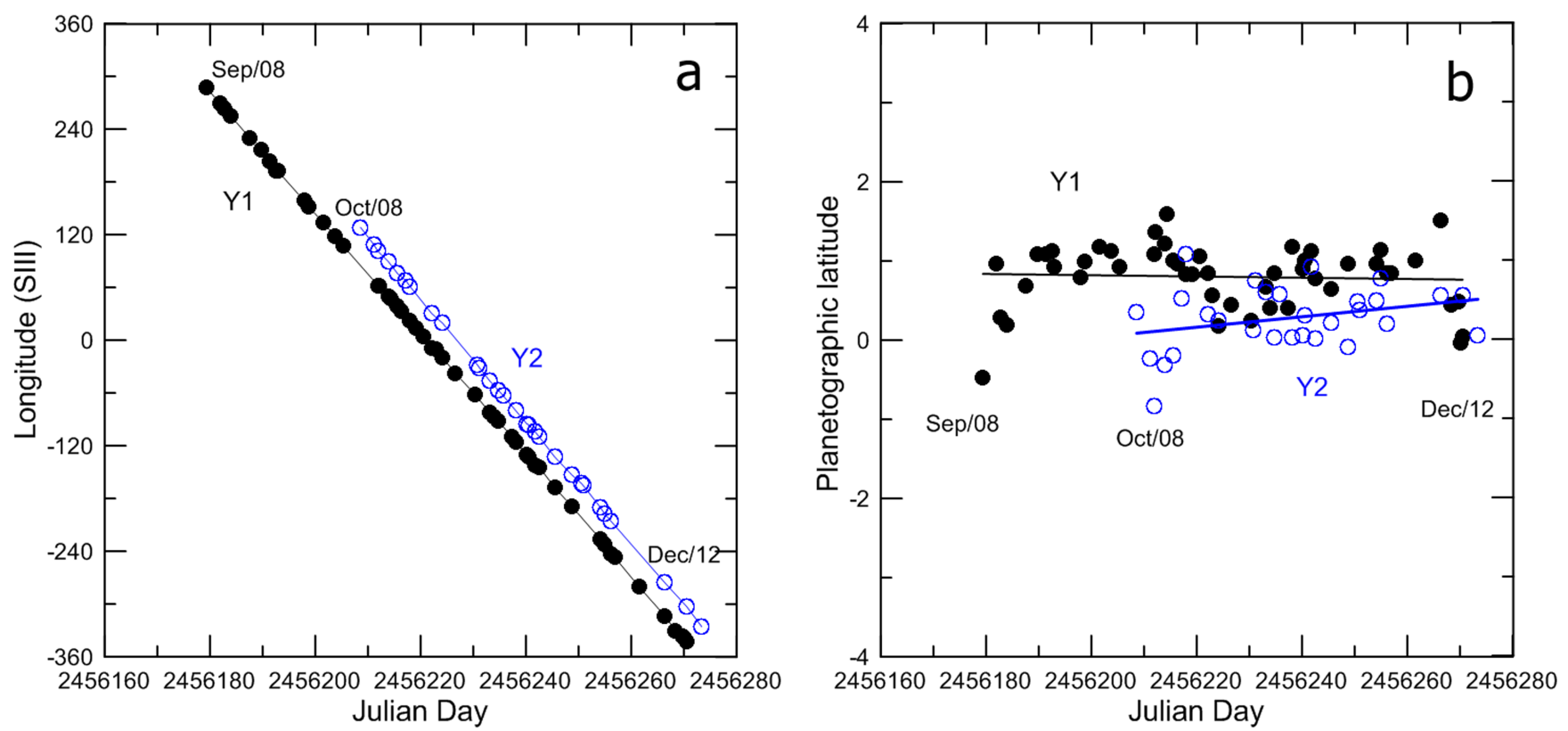

Fig. 3. Motions of Y1 and Y2 between September and December 2012: a) drift in System III Longitude; b) latitude changes. The lines are a linear fit to the measured data.

algorithm that maximizes the cross-correlation of small sections of two images separated by a given temporal interval. Following Barrado-Izagirre et al. (2013), rather than performing a correlation of the images in terms of two-dimensional boxes, we modified the algorithm to perform a correlation of nearly zonal scans that are selected from the image. Each wind vector measurement is assigned to the central latitude of the correlation window. On the other hand, we performed a manual tracking of single cloud targets across the EZ.

In Fig. 7a, we present three zonal wind profiles for the equatorial region. One is the reference profile obtained with HST data from 1995 to 1998 (García-Melendo \& Sánchez-Lavega 2001) and the other two have been calculated using the above mentioned automatic correlation technique on the HST images for September 20 at the two available wavelengths: red $(763 \mathrm{~nm})$ and ultraviolet $(275 \mathrm{~nm})$. These wavelengths sense different altitude levels: upper haze $(275 \mathrm{~nm})$ and lower main cloud $(763 \mathrm{~nm})$. There is a good agreement between the 1995-1998 HST profile (García-Melendo \& Sánchez-Lavega 2001) and that measured at red wavelengths in 2012. However, there is a substantial difference between the wind profiles obtained in the red and UV with globally decreasing winds in UV. This is because of a vertical wind shear effect as shown by Li et al. (2006). The velocity of the Y1 and Y2 features, retrieved from the measurements shown in Fig. 3, are also represented and compared to the average zonal wind in Fig. 7. It is evident from this comparison that the $\mathrm{Y}$ features moved faster than the zonal wind at both levels: the upper haze level and the lower cloud level (Fig. 7b). The Y features moved approximately $20-40 \mathrm{~m} \mathrm{~s}^{-1}$ faster (eastward) relative to the mean zonal flow at the upper cloud level in the equator.

Because of the meridional wind shear across the equator $\left(\sim 10 \mathrm{~m} \mathrm{~s}^{-1}\right.$ on a meridional length $\left.\sim 4000 \mathrm{~km}\right)$, the ambient vorticity of the zonal flow is

$\zeta_{\mathrm{amb}}=\frac{\partial u}{\partial y} \approx \frac{\Delta u}{\Delta y} \approx \frac{10}{4 \times 10^{6}}=2.5 \times 10^{-6} \mathrm{~s}^{-1}$.

Therefore, any dynamically non-coherent feature would be destroyed in about $t=\zeta_{\mathrm{amb}}^{-1} \approx 5$ days. Similarly, assuming that the difference between the wind profiles at red and UV wavelengths is due to vertical shear of the wind across a scale height $(H=$ $20 \mathrm{~km}$ ), and taking a mean velocity difference between these two levels $\Delta u \sim 14 \mathrm{~m} \mathrm{~s}^{-1}$ we obtain

$\frac{\partial u}{\partial z} \approx \frac{\Delta u}{\Delta z} \approx 7 \times 10^{-4} \mathrm{~s}^{-1}$,

giving a characteristic time for a feature being destroyed by the shear in $\sim 0.4 \mathrm{~h}$. The Y's long lifetime and survival against wind shears means that they are coherent stable features.

Related to its vertical structure, Fig. 8 shows a particular observation in which one of the $\mathrm{Y}$ features is apparently moving above the clouds detected at red wavelengths, suggesting that it is located above the ammonia cloud tops but without reaching the upper haze sensed at UV. Accordingly, the dark clouds forming the Y structure might be located somewhere between the lower ammonia cloud and the upper ultraviolet absorbing hazes.

\section{Shallow water simulations on Jupiter's equator}

The Y1 and Y2 features kept their coherence for weeks in a wind sheared region, where they were confined in latitude and moving faster than their surrounding clouds. The above properties favour the interpretation of the Y phenomenon as a wave. To test this hypothesis, we performed non-linear shallow water (SW) simulations of Jupiter's equatorial atmosphere. These SW models, although physically simple, capture some of the most important dynamical properties of the atmosphere. These models have been successfully used in many situations for the giant planets (Vasavada \& Showman 2005) and represent a frame to interpret wave dynamics of Earth's equatorial atmosphere, as in the case of the Matsuno-Gill model (Matsuno 1966; Gill 1980). We run one- and two-layer simulations, as described in GarcíaMelendo \& Sánchez-Lavega 2016). The one-layer SW model is the simplest representation, which allows us direct control over the response of the Jovian atmosphere to a disturbance, thereby avoiding long computing times. Two-layer SW models are, on the other hand, the simplest approach to a stratified atmosphere; 

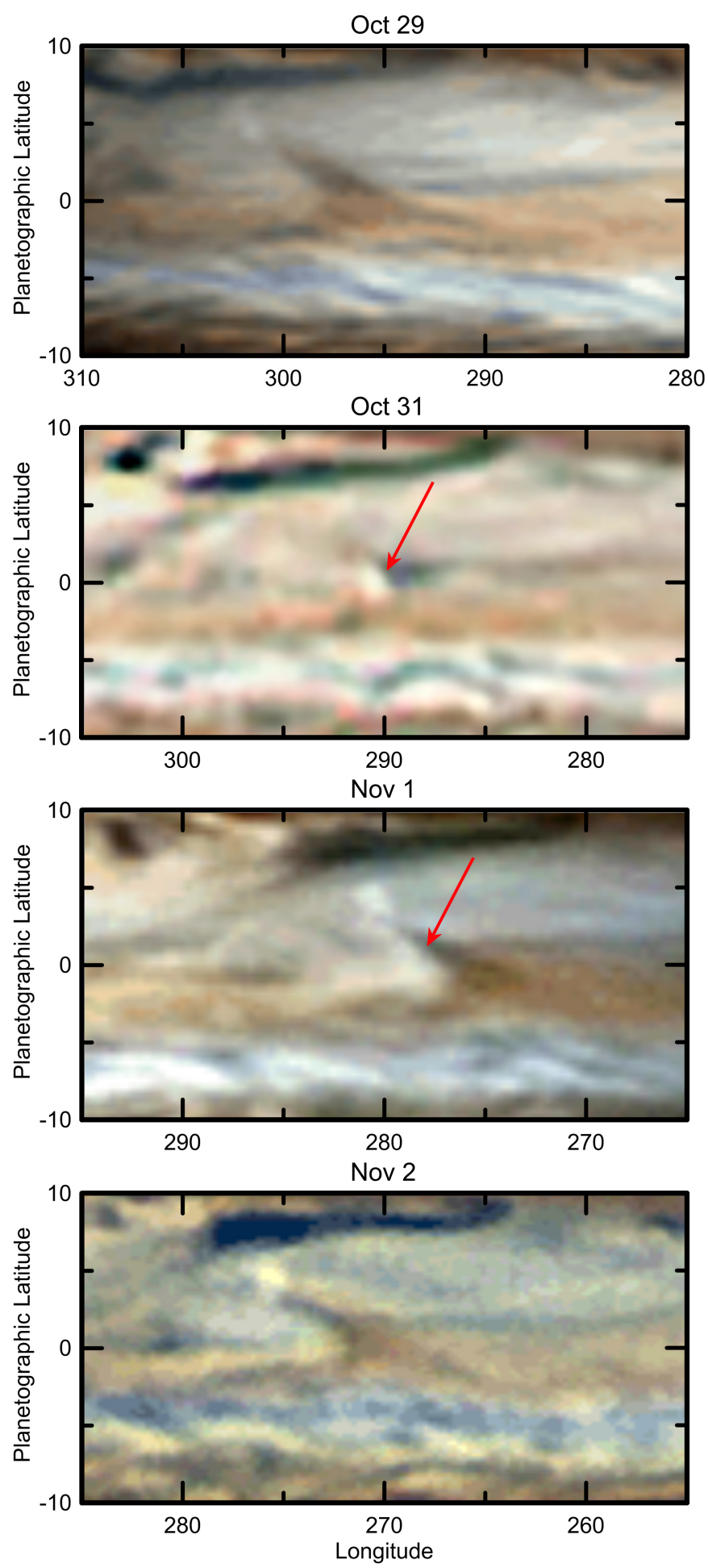

Fig. 4. Maps at the indicated dates in 2012 showing rapid changes related to bright clouds (indicated by red arrows) at the centre of the "V" vertex of the Y features. Image authors from top to bottom: C. Go, J. J. Poupeau, T. Akutsu, and F. Willems.

in this case, we can emulate the troposphere-stratosphere interface, and compare results with those yielded by the simpler onelayer model. In particular, linear theory tells us that two-layer SW models can behave according to two different dynamical modes: the barotropic and baroclinic modes (Gill 1982). In the barotropic mode, the system is identical to a single-layer model where the equivalent layer thickness is just the sum of the two,
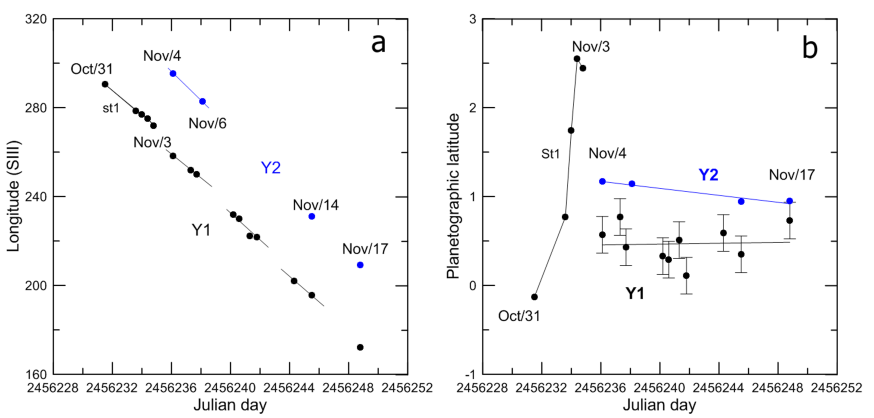

Fig. 5. Motions of bright spots related to $\mathrm{Y} 1$ and $\mathrm{Y} 2$ features in October and November 2012: a) drift in System III Longitude; b) latitude changes. The lines are linear fits to the measured data for tracked clouds. Bright feature $S t 1$ is indicated.
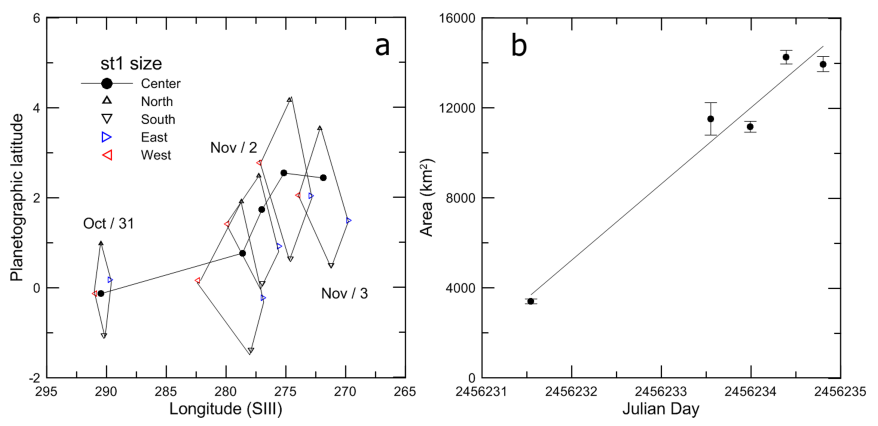

Fig. 6. a) Size evolution of bright feature $S t 1$ estimated from cardinal points north-south-east-west; b) area evolution for bright feature $S t 1$ with the line representing a linear fit to the data points.

upper $\mathrm{h}$ and lower $\mathrm{H}$ layer, thicknesses. In the baroclinic mode the stratification effect appears. When density contrast between the upper and lower layer, i.e.

$\rho^{\prime}=\frac{\rho_{1}-\rho_{2}}{\rho_{2}}=\ll 1$,

where subscripts 1 and 2 refer to the upper and lower layer, respectively, the system is dynamically equivalent to a one-layer system with equivalent gravity $g^{\prime}=g \rho^{\prime}$, and equivalent depth

$H_{\mathrm{e}}=\frac{h H}{h+H}$.

Our simulation domain consisted in a flat-bottomed (GarcíaMelendo \& Sánchez-Lavega 2016) rectangular $180^{\circ}$-long channel centred on the equator in the latitude interval $\left[+15^{\circ},-15^{\circ}\right]$ with a fixed longitudinal resolution of $0.25^{\circ} \mathrm{pix}^{-1}$. We imposed the continuous zonal flow measured by García-Melendo \& Sánchez-Lavega (2001) represented in the lower right panel in Fig. 2, which we switched on and off in the one layer case; in the two-layer version, zonal winds were always on. We disturbed the system with a Gaussian-shaped elevation $\eta$ on the free surface centred at the equator $\left(\varphi=0^{\circ}\right)$. To avoid saturating the system with gravity waves, the perturbation was introduced by slowly increasing its amplitude, and extinguishing it to zero following an exponential law. Perturbation's amplitude, extension, and time during which it is active determine its intensity and the system's response. We established that reasonable forcing parameters were short lifetimes of between 10 and 15 days, including initial exponential amplitude increase and decay times; small maximum amplitudes of between 1 and $5 \mathrm{~m}$; a Gaussian shape $\sigma$ parameter for the free surface elevation of $1^{\circ}$ and a maximum radial perturbation extension of $1.5^{\circ}$. 

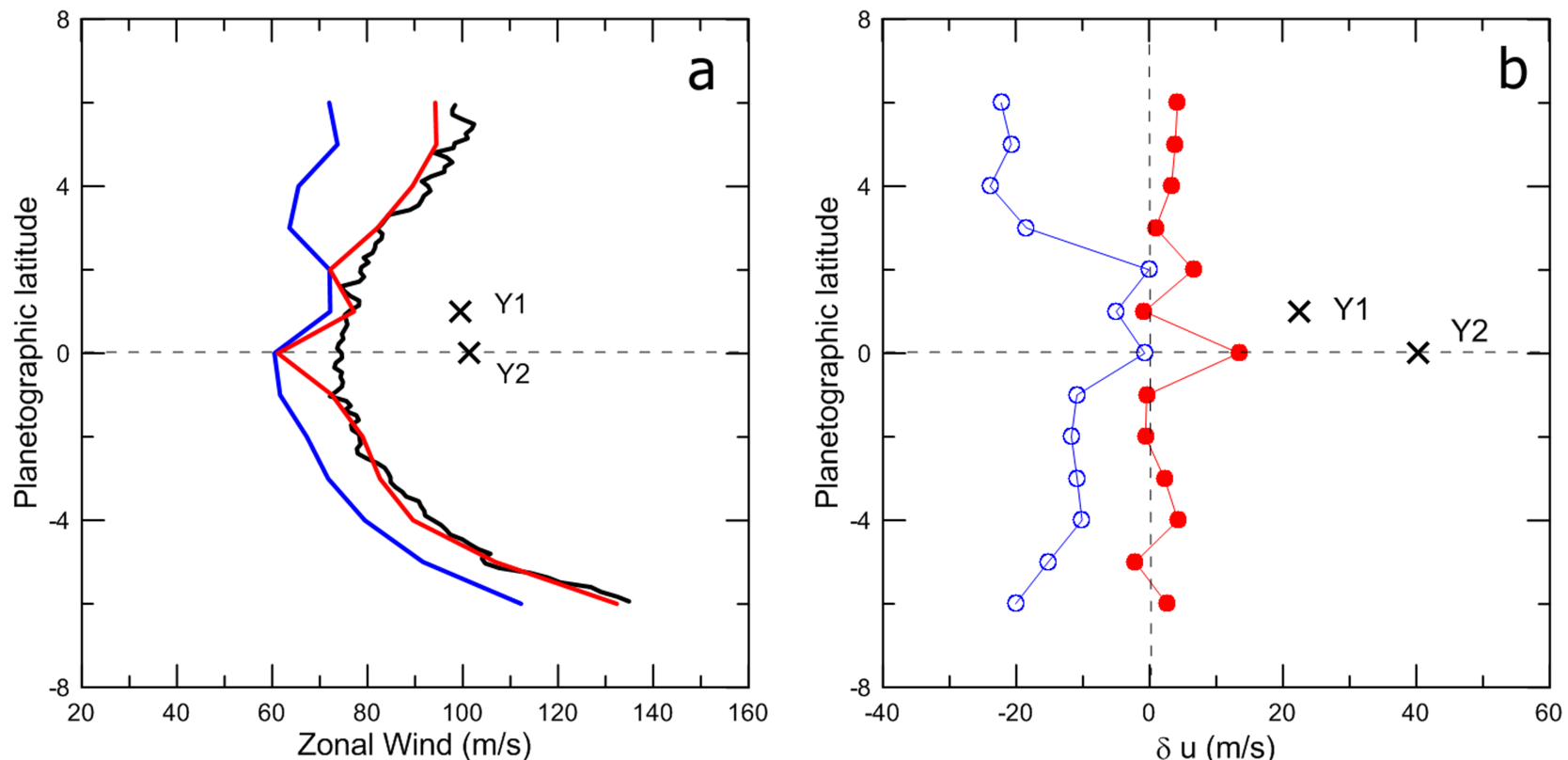

Fig. 7. Features velocities: a) zonal wind profiles HST data in September 2012 at two wavelengths: $763 \mathrm{~nm}$ (red line) and at $275 \mathrm{~nm}$ (blue line). The black line is a reference profile from García-Melendo \& Sánchez-Lavega (2001). The $\times$ are the measured speed of Y1 and Y2; b) differences in velocity between profiles. Blue line and dots: HST profile at $275 \mathrm{~nm}$ minus HST profile at $763 \mathrm{~nm}$ (indicates vertical wind shear); red line and dots: García-Melendo \& Sánchez-Lavega (2001) profile minus HST $763 \mathrm{~nm}$ profile (indicates long-term behaviour); the cross $(\times)$ represents the velocity difference between Y1 and Y2 relative to the $763 \mathrm{~nm}$ profile at their latitudes (which suggests wave phase speed for Y features).

Table 3. Range of parameters for single-layer SW numerical experiments, including a perturbation as described in the main text with a 15-day lifetime.

\begin{tabular}{ccc}
\hline \hline$H(\mathrm{~m})$ & $\eta(\mathrm{m})$ & $c_{\mathrm{K}}\left(\mathrm{m} \mathrm{s}^{-1}\right)$ \\
\hline 1000 & $3-200$ & 150 \\
500 & $3-200$ & 106 \\
200 & $3-100$ & 67 \\
100 & $3-5$ & 47 \\
50 & $3-5$ & 33 \\
20 & $3-5$ & 21 \\
10 & $1-2$ & 15 \\
5 & $1-2$ & 11 \\
\hline
\end{tabular}

Notes. The third column gives expected phase velocity $c_{\mathrm{K}}$ for a barotropic Kelvin wave travelling on the layer surface.

\subsection{The single-layer SW results}

For the one-layer case, Table 3 shows the range of the simulated parameters. When zonal winds are off (we assume that the atmosphere is at rest), the disturbance generates a typical pattern that we identify as formed by a pair of Rossby-Kelvin waves, such as those observed in Earth's equatorial atmosphere for nearly symmetric excitation (Matsuno 1966; Gill 1980), as shown in Fig. 9 (panel A). We obtain a Rossby wave and a Kelvin wave moving to the east as predicted by the theoretical $c_{\mathrm{k}}$. For trapped equatorial Kelvin waves, the phase velocity is $c_{\mathrm{K}}=\sqrt{g H}$ ( $g$ is gravity acceleration) and the meridional extension is $y_{\mathrm{K}}=\sqrt{2} L_{\mathrm{E}}$. Another important parameter in the system is the Rossby deformation radius $L_{\mathrm{E}}=\left(c_{\mathrm{K}} / \beta\right)^{1 / 2}$, where $\beta$ is the planetary vorticity gradient. When zonal winds are on, a similar pattern emerges, but this time phase speeds add to the background zonal flow velocity. In the case illustrated in Fig. 9, panel B, the Kelvin wave propagates at $\sim 187 \mathrm{~m} \mathrm{~s}^{-1}$; this is not its phase speed, but is the result after adding $c_{\mathrm{K}}$ to the local zonal flow speed of $80 \mathrm{~m} \mathrm{~s}^{-1}$.
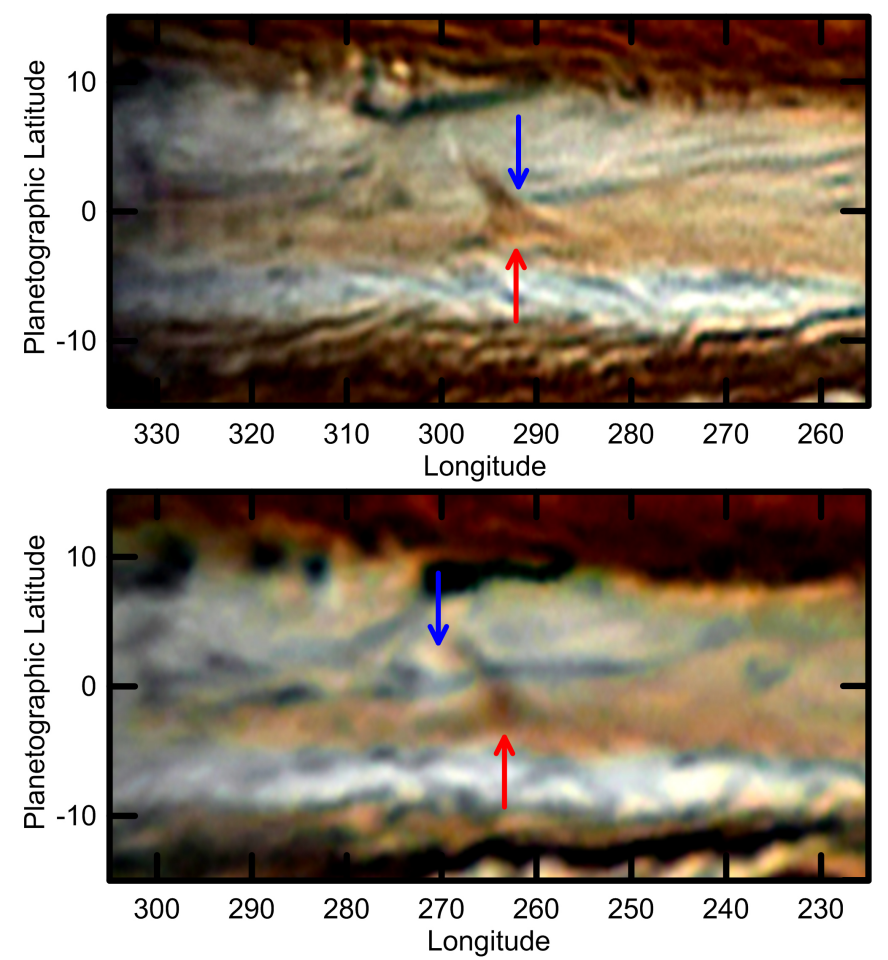

Fig. 8. A pair of RGB image composites in October 29 (top) and November 3 (bottom) showing the apparent motion of feature Y1 (indicated with a red arrow) over a background linear cloud (indicated with a blue arrow).

In both cases, with and without background zonal flow, the symmetric lobules of the Rossby wave propagate westward relative to the excitation source, whereas the single Kelvin wave propagates eastward. For sufficiently long-time simulations, the 

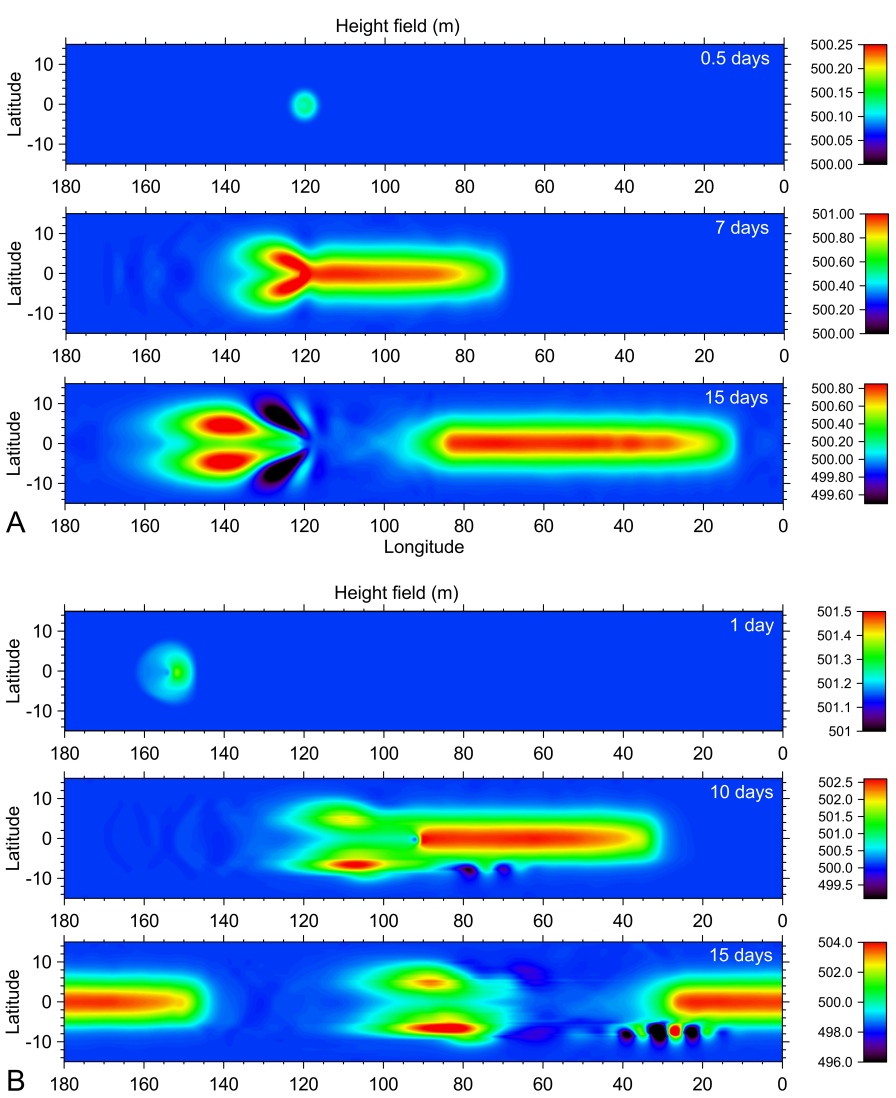

Fig. 9. A) Surface topography map of one-layer SW simulations at the equatorial region under Jovian conditions when zonal winds are turned off and disturbance amplitude is $3 \mathrm{~m}$ and $L_{\mathrm{E}}=5000 \mathrm{~km}$. A pair of Rossby-Kelvin wave forms resembling the Matsuno-Gill model. From the point of view of the injected pulse, Rossby waves (double symmetric lobe at left) drift to the west,while the Kelvin wave (large bar at right) moves to the east $\left(c_{\mathrm{K}}=108 \mathrm{~m} \mathrm{~s}^{-1}\right)$. B) Surface topography map for the same conditions as in panel $\mathbf{A})\left(L_{\mathrm{E}}\right.$ and initial perturbation amplitude), when zonal winds are switched on. In this case $c_{\mathrm{K}}=187 \mathrm{~m} \mathrm{~s}^{-1}$, which is very close to the sum of background zonal wind speed and theoretical velocity phase for the Kelvin wave.

Kelvin and Rossby waves separate and encounter each other after travelling the whole domain in opposite directions. Assuming then that surface elevation represents cloud albedo of the Y feature, our simulation reproduces the $\mathrm{Y}$ pattern after a few days following the excitation. Although we can adjust $c_{\mathrm{K}}$ to the observed Y-drift velocities, the time evolution of the pattern resulting from the simulation does not correspond to Y1 and Y2, since the real Y structure was preserved in time. We can reduce layer thickness $H$ to control $c_{\mathrm{K}}$, but even for very thin layers (slow $c_{\mathrm{K}}$ ), Rosby and Kelvin waves separate so that we cannot reproduce a stable Y feature. The two-layer model is instrumental to obtain a more stable Y pattern.

\subsection{The two-layer SW results}

In the two-layer baroclinic mode, the theoretical Kelvin wave phase velocity is obtained from $c_{\mathrm{K}}^{\prime}=\sqrt{g^{\prime} H_{\mathrm{e}}}$. The parameter space explored in our two-layer model is given in Table 4. For this a range of values, $c_{\mathrm{K}}^{\prime}$ is between $3 \mathrm{~m} \mathrm{~s}^{-1}$ and $12 \mathrm{~m} \mathrm{~s}^{-1}$ and $L_{\mathrm{E}}^{\prime}=\sqrt{c_{\mathrm{K}}^{\prime} / \beta}$ ranges from $1000 \mathrm{~km}$ to $1600 \mathrm{~km}$. Figure 10 shows a simulation producing a coherent $\mathrm{Y}$ feature in the interface surface between the two layers for the baroclinic mode when zonal winds are on. The initial disturbance generates a
Table 4. Parameter used in the two-layer shallow water model.

\begin{tabular}{cccccc}
\hline \hline$h(\mathrm{~m})$ & $H(\mathrm{~m})$ & $\eta(\mathrm{m})$ & $\rho_{1}\left(\mathrm{~kg} \mathrm{~m}^{-3}\right)$ & $\rho_{2}\left(\mathrm{~kg} \mathrm{~m}^{-3}\right)$ & $c_{\mathrm{K}}^{\prime}\left(\mathrm{m} \mathrm{s}^{-1}\right)$ \\
\hline $20,10,5$ & 1000 & $5,3,2,1$ & 0.1 & $0.105-0.15$ & $4.6-12.1$ \\
$20,10,5$ & 500 & $3,2,1.5,1$ & 0.1 & 0.15 & 12.0 \\
20 & 100 & $5,3,2$ & 0.1 & $0.105-0.15$ & $4.2-11.2$ \\
20 & 50 & $5,3,2$ & 0.1 & $0.105-0.15$ & $3.9-10.3$ \\
20 & 20 & $5,3,2$ & 0.1 & $0.105-0.15$ & $3.3-8.7$ \\
20 & 10 & 2,1 & 0.1 & $0.105-0.15$ & $2.7-7.1$ \\
\hline
\end{tabular}

Rossby-Kelvin pair similar to the one-layer case, but now the pair is confined to a narrower equatorial region and expands zonally with a more complex shape. In Fig. 10 the Kelvin wave has a positive phase velocity propagating eastward with a total phase velocity of $\sim 82 \mathrm{~m} \mathrm{~s}^{-1}$. By subtracting the background zonal velocity at the equator, where zonal speed is $\sim 75 \mathrm{~m} \mathrm{~s}^{-1}$, the simulated phase speed is $\sim 7 \mathrm{~m} \mathrm{~s}^{-1}$, which is close to the theoretical value of $\sim 5 \mathrm{~m} \mathrm{~s}^{-1}$. The meridional excursion of this equatorially trapped wave is consistent with a theoretical value of $y_{\mathrm{K}}^{\prime}=\sqrt{2} L_{\mathrm{E}}^{\prime} \approx 1400 \mathrm{~km}=1.2^{\circ}$. The Rossby wave has a negative (westward) phase velocity given by

$c_{\mathrm{R}}^{\prime}=-\frac{c_{\mathrm{K}}^{\prime}}{2 n+1}$,

where $n$ is the wave mode $(n=1,2, \ldots)$. Both Rossby $(n=1)$ and Kelvin waves are similar to those obtained by many authors for the terrestrial equatorial ocean in response to heat and wind forcing (Philander \& Pacanowski 1984), where the tropical thermocline acts as a waveguide for planetary-scale equatorially trapped waves.

The best outcomes resulted for thin upper layer thicknesses $h$ over high $H$ values and low density contrast. Our two-layer SW model simulations generate, in the baroclinic mode, a stable perturbation resembling the Y-like feature for more than two months, which mimics the observed Y morphology. Results suggest that the feature formed in a very thin layer. The common edge of the Rossby and Kelvin waves must move approximately at the same speed for the Y feature to be coherent. For the particular simulation in Fig. 9, the drift rate in longitude of the common point for the Rossby and Kelvin waves is about $-5.0^{\circ}$ day $\left(\sim 75 \mathrm{~m} \mathrm{~s}^{-1}\right)$, which is somewhat slower than the observed $-6.9^{\circ} /$ day $\left(100 \mathrm{~m} \mathrm{~s}^{-1}\right)$ value (Fig. 3$)$.

In both, one-layer and two-layer models, a pattern of shortwavelength Rossby waves is excited in the peak of the jet stream at $7^{\circ} \mathrm{S}$, which resembles the pattern observed at this latitude; this was reported in previous works such as García-Melendo et al. (2011). The $7^{\circ} \mathrm{S}$ jet violates the Rayleigh-Kuo barotropic instability criteria and is highly unstable under any small perturbation, resulting in an independent phenomenon from the RossbyKelvin equatorial wave pair.

\section{Discussions and conclusions}

We have observed and characterized a peculiar cloud structure in the equator of Jupiter, which we call the Y features. Two of them were simultaneously present between September and December 2012 and developed occasionally short-lived bright spots at the vertex of the Y structure. The properties of these features suggest they are a manifestation of an equatorially trapped Rossby-Kelvin wave. Simulations of the disturbed surface elevation in one- and two-layer shallow water models under Jovian conditions are able to reproduce the main characteristics of the 

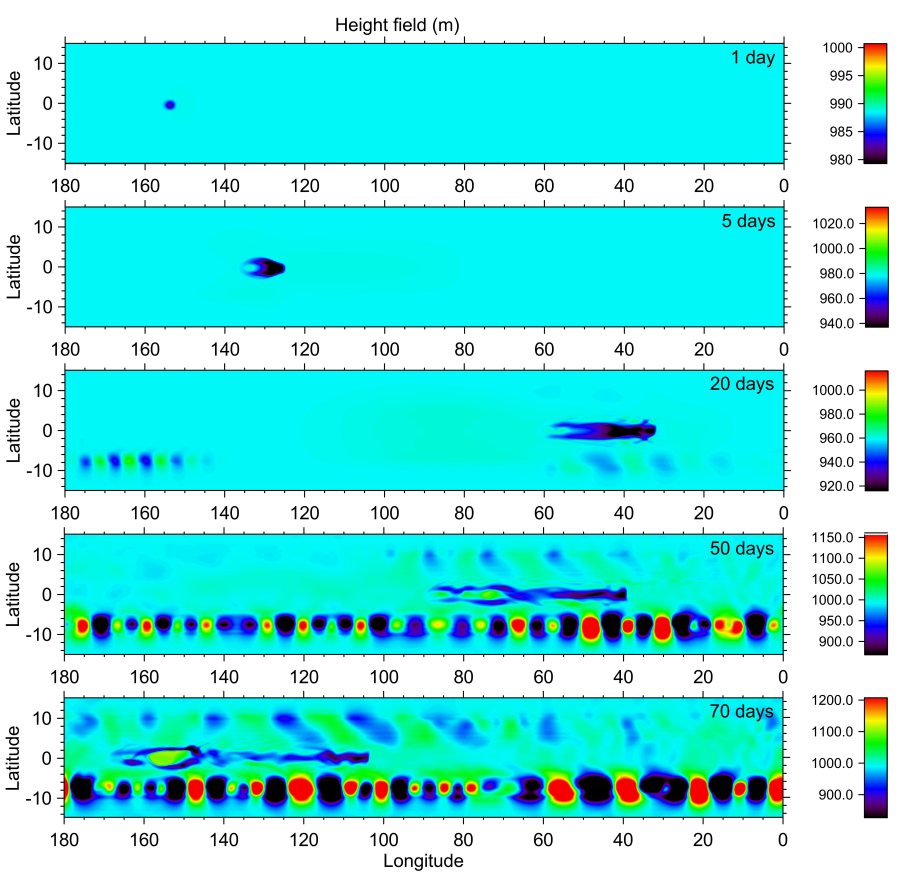

\section{$1000.0-$
980.0
$960.0-$
940.0
920.0}

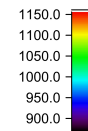

Fig. 10. Simulations of the $\mathrm{Y}$ feature under a two-layer SW model when zonal winds are switched on. The interface surface topography is shown. Data for this simulation: $h=20 \mathrm{~m}, H=1000 \mathrm{~m}, \rho_{1} / \rho_{2}=0.95$, and disturbance amplitude $\eta=3 \mathrm{~m} .\left(c_{\mathrm{K}}=7 \mathrm{~m} \mathrm{~s}^{-1}\right)$.

Y morphology. The SW models are simple physical models, and therefore we do not intend to exactly reproduce all the observed properties of the waves. Rossby-Kelvin waves are known to be present in Earth's equator, where they play an important role in the atmosphere in the quasi-Biennial oscillation (Lindzen \& Holton 1968; Holton \& Lindzen 1972) and in the oceans in the El Niño Southern Oscillation (Philander \& Pacanowski 1984). Particularly, the pair we have described is a classical picture of the well-known Matsuno-Gill problem (Matsuno 1966; Gill 1980). Similarly, our simulations generate a Rossby-Kelvin pair under Jovian conditions. We have not explored the possibility that the Y features correspond to other wave types, such as nonlinear solitary waves of the type proposed by Maxworthy et al. (1978), Maxworthy \& Redekopp (1980) for the Great Red Spot and other close features. The fact that the solitary wave solutions to the $\mathrm{KdV}$ equation in those works involved elliptical shape features due to combined D-E cyclonic-anticyclonic wave pairs seems to be at odds with the observed shape of the Y feature. However, the non-dispersive character observed for the $\mathrm{Y}$ feature agrees with a solitary wave behaviour. Because of the lack of data, we leave this hypothesis for future exploration in case that other Y structures form and are studied with more detail. The origin of the brightening clouds, occasionally observed in the vertex of the $\mathrm{Y}$ feature, which we identify with the centre of the Rossby wave part (Figs. 4 to 6), is intriguing. The area expansion, rapid evolution, and high albedo of these clouds points towards the condensation on ascending motions that inject unpolluted ammonia ice at the Y-level cloud. Since the estimated speeds are lower than those typical of moist convection (Gierasch et al. 2000; Hueso \& Sánchez-Lavega 2001), we can speculate that the updrafts could be due to the ascending motions in the internal branch of the Rossby wave.

Future observations of Jupiter's equator will serve to characterize the wave excitation mechanisms, wave life cycle, persistence, and interactions to better constraint their properties in order to develop more advanced models.
Acknowledgements. This work was supported by the Spanish MICIIN AYA2012-36666 with FEDER support, Grupos Gobierno Vasco IT765-013, and UPV/EHU UFI11/55. This work is partly based on observations obtained from the data archive at the Space Telescope Science Institute. STScI is operated by the Association of Universities for Research in Astronomy, Inc., under NASA Contract NAS 5- 26555. The IOPW contributors are from the International Outer Planet Watch Team IOPW-PVOL: http://www. pvol . ehu.es/pvol/index. jsp?action=iopw.

\section{References}

Allison, M. 1990, Icarus, 83, 282

Arregi, J., Rojas, J. F., Sánchez-Lavega, A., \& Morgado, A. 2006, J. Geophys. Res., 111, 9010

Arregi, J., Rojas, J., Hueso, R., \& Sánchez-Lavega, A. 2009, Icarus, 202, 358

Asay-Davis, X. S., Marcus, P. S., Wong, M. H., \& de Pater, I. 2011, Icarus, 211, 1215

Barrado-Izagirre, N., Sánchez-Lavega, A., Pérez-Hoyos, S., \& Hueso, R. 2008, Icarus, 194, 173

Barrado-Izagirre, N., Rojas, J. F., Hueso, R., et al. 2013, A\&A, 554, A74

Cano, J. 1998, in Grup d'Estudis Astronómics, Barcelona

Choi, D. S., Showman, A. P., Vasavada, A. R., \& Simon-Miller, A. A. 2013, Icarus, 223, 832

Deming, D., Reuter, D., Jennings, D., et al. 1997, Icarus, 126, 301

Flasar, F. M., \& Gierasch, P. J. 1986, J. Atm. Sci., 43, 2683

García-Melendo, E., \& Sánchez-Lavega, A. 2001, Icarus, 152, 316

García-Melendo, E., \& Sánchez-Lavega, A. 2016, Icarus, 1, submitted

García-Melendo, E., Arregi, J., Rojas, J. F., et al. 2011, Icarus, 211, 1242

Gierasch, P. J., Ingersoll, A. P., Banfield, D., et al. 2000, Nature, 403, 628

Gill, A. E. 1980, Quarterly J. Roy. Meteor. Soc., 106, 447

Gill, A. E. 1982, Atmosphere-Ocean Dynamics, 30 (San Diego, USA: Academic Press)

Holton, J. R., \& Lindzen, R. S. 1972, J. Atm. Sci., 29, 1076

Hueso, R., \& Sánchez-Lavega, A. 2001, Icarus, 151, 257

Hueso, R., Legarreta, J., García-Melendo, E., Sánchez-Lavega, A., \& PérezHoyos, S. 2009, Icarus, 203, 499

Hueso, R., Legarreta, J., Pérez-Hoyos, S., et al. 2010, Planet. Space Sci., 58, 1152

Ingersoll, A. P., Dowling, T. E., Gierasch, P. J., et al. 2004, Dynamics of Jupiter's atmosphere, 105

Law, N. M., Mackay, C. D., \& Baldwin, J. E. 2006, A\&A, 446, 739

Li, L., Ingersoll, A. P., Vasavada, A. R., et al. 2006, J. Geophys. Res., 111, 4004

Limaye, S. S. 1986, Icarus, 65, 335

Lindzen, R. S., \& Holton, J. R. 1968, J. Atm. Sci., 25, 1095

Matsuno, T. 1966, J. Meteorol. Soc. Japan. Ser. II, 44, 25

Maxworthy, T., \& Redekopp, L. G. 1980, Science, 210, 1350

Maxworthy, T., Redekopp, L. G., \& Weidmann, P. D. 1978, Icarus, 33, 388

Mendikoa, I., Pérez-Hoyos, S., \& Sánchez-Lavega, A. 2012, EPJP, 33, 1611

Ordóñez-Etxeberria, I., del Río-Gaztelurrutia, T., \& Sánchez-Lavega, A. 2014, EPJ, 35, 045020

Ortiz, J. L., Orton, G. S., Friedson, A. J., et al. 1998, J. Geophys. Res., 103, 23051

Peralta, J., Sánchez-Lavega, A., López-Valverde, M. A., Luz, D., \& Machado, P. 2015, Geophys. Res. Lett., 42, 705

Philander, S. G. H., \& Pacanowski, R. C. 1984, Geophys. Res. Lett., 11, 802

Porco, C. C., West, R. A., McEwen, A., et al. 2003, Science, 299, 1541

Reuter, D. C., Simon-Miller, A. A., Lunsford, A., et al. 2007, Science, 318, 223

Rogers, J. H. 1995, The giant planet Jupiter (Cambridge; New York, NY, USA: Cambridge University Press)

Sánchez-Lavega, A., \& Gómez, J. M. 1996, Icarus, 121, 1

Sánchez-Lavega, A., Orton, G. S., Hueso, R., et al. 2008, Nature, 451, 437

Sánchez-Lavega, A., Orton, G. S., Hueso, R., et al. 2011, Icarus, 214, 462

Sánchez-Lavega, A., Pérez-Hoyos, S., Hueso, R., del Río-Gaztelurrutia, T., \& Oleaga, A. 2014, Eur. J. Eng. Education, 39, 518

Schubert, G. 1983, General circulation and the dynamical state of the Venus atmosphere, eds. D. M. Hunten, L. Colin, T. M. Donahue, \& V. I. Moroz, 681

Simon, A. A. 1999, Icarus, 141, 29

Simon-Miller, A. A., Poston, B. W., Orton, G. S., \& Fisher, B. 2007, Icarus, 186, 192

Simon-Miller, A. A., Rogers, J. H., Gierasch, P. J., et al. 2012, Icarus, 218, 817

Vasavada, A. R., \& Showman, A. P. 2005, Rep. Progr. Phys., 68, 1935

Yamazaki, Y., Read, P., \& Skeet, D. 2005, Planet. Space Sci., 53, 508 , 